\title{
Enhanced-Transient Expression of Hepatitis CVirus Core Protein in Nicotiana tabacum, a Protein With Potential Clinical Applications
}

\author{
Sara Mohammadzadeh ${ }^{1}$; Alireza Khabiri ${ }^{2}$; Farzin Roohvand ${ }^{3,{ }^{*}}$; Arash Memarnejadian ${ }^{4}$; Ali \\ Hatef Salmanian ${ }^{5}$; Soheila Ajdary ${ }^{6}$; Parastoo Ehsani ${ }^{1, *}$ \\ ${ }^{1}$ Department of Molecular Biology, Pasteur Institute of Iran, Tehran, IR Iran \\ ${ }_{2}^{2}$ Department of Mycology, Pasteur Institute of Iran, Tehran, IR Iran \\ 3 Department of Virology, Pasteur Institute of Iran, Tehran, IR Iran \\ 4 Department of Virology, Pasteur Institute of Iran, Tehran, IR Iran \\ 5 Department of Hepatitis and AIDS, Pasteur Institute of Iran, Tehran, IR Iran
5 Department of Plant Biotechnology, National Institute of Genetic Engineering and Biotechnology, Tehran, IR Iran \\ 6 Department of Immunology, Pasteur Institute of Iran, Tehran, IR Iran \\ *Corresponding Author:Parastoo Ehsani, Department of Virology, Pasteur Institute of Iran, Tehran, IR Iran. Tel/Fax.:+98 21 6411-2167, E-mail:p_ehsani@pasteur.ac.ir. Farzin Roohvand, \\ Department of Virology, Pasteur Institute of Iran, Tehran, IR Iran. Tel/Fax:+98-2166496682, E-mail: rfarzin@pasteur.ac.ir. \\ Received: May 25, 2014; Revised: September 29, 2014; Accepted: October 23, 2014
}

\begin{abstract}
Background: Hepatitis C virus (HCV) is major cause of liver cirrhosis in humans. HCV capsid (core) protein (HCVcp) is a highly demanded antigen for various diagnostic, immunization and pathogenesis studies. Plants are considered as an expression system for producing safe and inexpensive biopharmaceutical proteins. Although invention of transgenic (stable) tobacco plants expressing HCVcp with proper antigenic properties was recently reported, no data for "transient-expression" that is currently the method of choice for rapid, simple and lower-priced protein expression in plants is available for HCVcp.

Objectives: The purpose of this study was to design a highly codon-optimized HCVcp gene for construction of an efficient transient-plant expression system for production of HCVcp with proper antigenic properties in a regional tobacco plant (Iranian Jafarabadi-cultivar) by evaluation of different classes of vectors and suppression of gene-silencing in tobacco.

Materials and Methods: A codon-optimized gene encoding the Kozak sequence, 6xHis-tag, HCVcp (1-122) and KDEL peptide in tandem (from N-to C-terminal) was designed and inserted into potato virus-X (PVX) and classic pBI121 binary vectors in separate cloning reactions. The resulted recombinant plasmids were transferred into Agrobacterium tumefaciens and vacuum infiltrated into tobacco leaves. The effect of gene silencing suppressor P19 protein derived from tomato bushy stunt virus on the expression yield of HCVcp by each construct was also evaluated by co-infiltration in separate groups. The expressed HCVcp was evaluated by dot and western blotting and ELISA assays.

Results: The codon-optimized gene had an increased adaptation index value (from 0.65 to 0.85 ) and reduced GC content (from 62.62 to 51.05) in tobacco and removed the possible deleterious effect of "GGTAAG" splice site in native HCVcp. Blotting assays via specific antibodies confirmed the expression of the $15 \mathrm{kDa}$ HCVcp. The expression level of HCVcp was enhanced by $4-5$ times in P19 co-agroinfiltrated plants with better outcomes for PVX, compared to pBI121 vector (0.022\% versus $0.019 \%$ of the total soluble protein). The plant-derived HCVcp (pHCVcp) could properly identify the HCVcp antibody in HCV-infected human sera compared to Escherichia coli-derived HCVcp (eHCVcp), indicating its potential for diagnostic/immunization applications.

Conclusions: By employment of gene optimization strategies, use of viral-based vectors and suppression of plant-derived gene silencing effect, efficient transient expression of HCVcp in tobacco with proper antigenic properties could be possible.
\end{abstract}

Keywords:Hepatitis C Virus Core; Transgenic; Transient Expression; Tobacco

\section{Background}

Hepatitis C virus (HCV), the major cause of blood-borne chronic hepatitis with potential progression to cirrhosis, has infected around 170 million people globally(1). Due to heterogeneity of HCV proteins, their high mutation rates and complex pathogenesis, no approved vaccine for human application against this viral infection is available to date $(2,3)$. Recognition of conserved epitopes in HCV proteins and advancements in the formulation of vaccines in novel modalities, however, have led to the placement of a few HCV vaccines in the pipeline of human clinical trials in recent years $(4,5)$.

HCV holds a single-stranded positive-sense RNA genome which encodes for three structural (core protein and envelope proteins E1 and E2) and six nonstructural proteins (4). Among the HCV proteins, core (HCVcp) is the most conserved HCV antigen, and hence, a good candidate to be considered for HCVvaccine formulations (4-6). Accordingly, application of even isolated HCVcp-CTL epitopes (810 amino acids) in the context of synthetic multi-epitope $\mathrm{HCV}$ vaccines has also been reported (7-9). Besides, anticore antibodies are the first to be raised after the onset of infection, a property that has provided an important diagnostic value for this protein and has located it among antibody-capturing antigens in commercially available

Copyright (C) 2014, Kowsar Corp. This is an open-access article distributed under the terms of the Creative Commons Attribution-NonCommercial 4.0 International License (http://creativecommons.org/licenses/by-nc/4.0/) which permits copy and redistribute the material just in noncommercial usages, provided the original work is properly cited. 
serological assays for HCV diagnosis (10). In addition, nucleotide sequence of HCVcp is supposed to encode for other alternated frame-shift proteins (ARFPs) with important pathogenic roles in chronic HCV infection and cirrhosis $(4,11)$. Therefore, to fulfill different diagnostic, research and therapeutic demands, production of HCVcp in various expression systems has been addressed (4-5). However, since the C-terminal hydrophobic region of HCVcp exerts immune-suppressive effects $(4,12)$, its first $120 \mathrm{~N}$-terminal residues, the hydrophilic region (HCVcp $\mathrm{N}-120$ ), which contains both nuclear localization signals (NLS) (13) and most of the conserved B and T cell epitopes, is usually employed for different diagnostic $(10,14)$ and vaccine applications (15-17).

Plants have an extensive potential to provide safe and inexpensive sources of biopharmaceutical and vaccination proteins (18). Currently, several plant-derived viral proteins such as hepatitis B surface antigen (19) and Norwalk virus capsid protein (20) are in vaccination clinical trials. Recently, invention of transgenic tobacco plants expressing HCVcp stably at T0 and T1 generations (21) or directing the expression of its 143-amino acid Nterminal in tobacco chloroplasts (22) were reported. Although these prior reports proved the proper antigenic structure of the plant-derived HCVcp for diagnostic purposes and indicated its full functionality to react with poly/monoclonal anti-core antibodies and HCV-infected human sera, however, both studies addressed only the transgenic (stable) tobacco plant generation for HCVcp. However, transient expression in plants, compared with transgenic plant generation, is currently the method of choice due to several advantages like the simplicity and feasibility of rapid protein expression, omitting tissue culture and regeneration costs and ease of large scale industrial plans (23-25). An important example of transient expression in plants is the production of seasonal influenza vaccines in tobacco (26). The success in efficient plant transient expression, however, depends on several factors such as: codon-optimization of the heterologous gene according to the plant-codon adaptation index (27, $28)$, selection of the proper expression vector $(29,30)$, and efficient inhibition of the gene silencing phenomenon which suppresses the expression of foreign genes in plants (31). While at present knowledge and techniques for the abovementioned parameters are being improved, providing novel data on transient expression process for the regionally-adapted plant hosts in general, and overcoming the plant-based gene silencing phenomenon in specific, is of high importance.

\section{Objectives}

In this study, we aimed to: i) Construct an efficient transient tobacco expression system for HCVcp N-121 by designing a highly codon-optimized gene and employment of the Iranian Jafarabadi-tobacco plant cultivar which is a high biomass producer ii) Evaluate the expression level of HCVcp for a potato virus X-based vector (PVX) (32) com- pared to a classic pBI121 binary plant vector in co-agroinfiltration with P19 gene silencing suppressor plasmid and iii) Assess the antigenicity of this tobacco-derived HCVcp for potential clinical (diagnostic and vaccine formulation) applications.

\section{Materials and Methods}

\subsection{Codon Optimization of HCVcp Gene and Con- struction of Expression Vectors}

The pIVEX2.4a core plasmid encoding HCVcp N-121, used as both the source of the sequence for construction of plant-based expression vectors and for the expression of $\mathrm{N}$-terminally 6xHis-tagged HCVcp protein in BL21-AI strain of Escherichia coli by arabinose induction (as positive control) was previously described $(33,34)$. Several modifications for optimized (plant) codon-usage of HCVcp nucleotide sequence were considered, including: i) Codon optimization according to the codon adaptation index of nuclear-encoded genes of tobacco (35), ii) Removal of (plant) mRNA destabilizing sequences from the native HCVcp coding sequence, iii) Addition of Kozak (GCCACCATGGC) sequence (36) and hexahistidine (6xHis)-tag for nickel affinity purification at the $5^{\prime}$ site, iv) Addition of nucleotides encoding endoplasmic reticulum retrieval signal (KDEL) at the $3^{\prime}$ end (37), v) Addition of BamHI and SacI restriction sites at both ends of the gene for directional cloning into the same sites of the plant expression binary vector pBI121 (Figure 1). The plant-optimized HCVcp gene for recombinant expression in tobacco (also termed $\mathrm{Tr}$-HCVcp) was synthesized and delivered as a clone in pUC57 plasmid by ShineGene Molecular Biotech Inc. (Shanghai, China). As shown in Figure $3 \mathrm{~A}$, the synthetic gene was subcloned into BamHI and SacI sites of the pBI121 vector (38) under the control of CaMV $35 \mathrm{~S}$ promoter and upstream of the nopaline synthase transcriptional terminator (NOS-Ter). In this study, the PVX-GW vector (32), which was kindly gifted by Dr. Cristiano Lacorte (EMBRAPA Recursos Geneticos e Biotecnologia, Brasília, Brazil), was used as the PVX-based viral vector. For cloning of the Tr-HCVcp sequence into PVX-GW vector, the synthetic gene was PCR amplified using a forward primer, F-Kozak-VX: 5'-TAATATCGATCTCGAGCCACCATGGCTCATCACC-3' and a reverse primer, R-core-VX: 5'-TAATGTCGACGGATCCTCAGAGTTCGTCCTTCTTTC-3' harboring ClaI and SalI restriction sites (underlined sequences). PCR amplification was performed with 25 cycles at $94^{\circ} \mathrm{C}$ for 30 seconds, $58^{\circ} \mathrm{C}$ for 30 seconds, and $72^{\circ} \mathrm{C}$ for 90 seconds and $72^{\circ} \mathrm{C}$ for 15 minutes. The 439-bp amplicon was subsequently digested by ClaI and SalI enzymes and cloned into the same sites of PVX-GW vector under the control of duplicated PVX coat protein subgenomic promoter (CPP) (Figure $3 \mathrm{~B}$ ). All the recombinant constructs were confirmed by restriction and sequencing analyses using F-Kozak-VX and R-core-VX specific primers. All the cloning and molecular procedures were according to the standard protocols (39). 


\begin{tabular}{|c|c|c|c|c|c|c|c|c|c|c|c|c|c|c|c|c|}
\hline \multirow{3}{*}{$\begin{array}{l}\text { Optimal } \\
\text { original }\end{array}$} & \multicolumn{2}{|c|}{ BamHI } & & & & $\begin{array}{c}\mathrm{Al} \\
\mathrm{nce}\end{array}$ & & & & & \multirow{2}{*}{$\begin{array}{c}\text { H } \\
\text { CAT }\end{array}$} & \multirow{2}{*}{$\begin{array}{c}\mathrm{H} \\
\mathrm{CAC}\end{array}$} & \multirow{2}{*}{$\underset{\text { ATG }}{\mathbf{M}}$} & \multirow{2}{*}{$\underset{\text { AGt }}{\mathbf{S}}$} & \multirow{2}{*}{$\begin{array}{c}\mathbf{T} \\
\mathrm{ACa}\end{array}$} & \multirow{2}{*}{$\begin{array}{c}\text { I } \\
\text { ATT }\end{array}$} \\
\hline & $\overline{G G A}$ & & & S & & S & $\mathrm{CAT}$ & CAC & CAT & $\mathrm{CAC}$ & & & & & & \\
\hline & $\mathbf{P}$ & k & $\mathbf{P}$ & $c$ & $\mathbf{R}$ & $\mathbf{K}$ & $\mathbf{T}$ & $\mathbf{K}$ & $\mathbf{R}$ & $\mathbf{N}$ & $\mathbf{T}$ & $\mathbf{N}$ & R & $\begin{array}{c}\mathrm{AG} \\
\mathbf{R}\end{array}$ & $\begin{array}{c}\text { ACG } \\
\mathbf{P}\end{array}$ & $\begin{array}{c}\mathrm{AT} \\
0\end{array}$ \\
\hline ptimal & $\mathrm{CCt}$ & A & $\mathrm{CCa}$ & c & A & A & $\mathrm{ACC}$ & AAA & CGT & $\mathrm{AAC}$ & ACt & AAt & $\mathrm{aGa}$ & $\mathrm{aGg}$ & $\mathrm{cct}$ & $\mathrm{CA} a$ \\
\hline$r 1$ & $\mathrm{CCC}$ & $A A A$ & ССT & $\mathrm{CAA}$ & AGA & AAA & $\mathrm{ACC}$ & $A A A$ & CGT & $A A C$ & $\mathrm{ACC}$ & AAC & CGC & CGC & $\mathrm{CCA}$ & CAG \\
\hline & D & v & K & $\mathbf{F}$ & $\mathbf{P}$ & G & G & G & Q & I & V & G & G & v & $\mathbf{Y}$ & L \\
\hline pt & GAt & GTC & AAa & TTC & $\mathrm{CCa}$ & GGa & GGT & GGa & CAG & ATC & GTT & GGT & GGA & GTg & TAC & CTt \\
\hline 1 & GAC & GTC & AAG & TTC & CCG & GGC & GGT & GGT & CAG & ATC & GTT & GGT & GGA & GTT & TAC & CTG \\
\hline & I & P & $\mathbf{R}$ & $\mathbf{R}$ & G & $\mathbf{P}$ & $\mathbf{R}$ & L & G & v & $\mathbf{R}$ & $\mathbf{A}$ & $\mathbf{T}$ & $\mathbf{R}$ & K & $\mathbf{T}$ \\
\hline pt & TTG & CCt & CGt & AGa & GGt & $\mathrm{CCa}$ & AGG & cTt & GGa & GTG & $\mathrm{aGa}$ & GCt & ACT & AGG & AAG & $\mathrm{ACa}$ \\
\hline or: & TTG & CCG & CGC & AGG & ; GGC & $\mathrm{CCC}$ & AGG & TTG & GGT & GTG & CGC & GCG & ACT & AGG & AAG & ACT \\
\hline & $\mathbf{S}$ & L & $x$ & $\mathbf{S}$ & $x$ & $\mathbf{P}$ & $\mathbf{R}$ & $\checkmark$ & 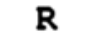 & 2 & Q & P & I & $\mathbf{P}$ & $\mathbf{K}$ & $\boldsymbol{A}$ \\
\hline$p t$ & TCt & GAG & $\mathrm{CAa}$ & $\mathrm{TCa}$ & CAg & ССТ & $\mathrm{aGa}$ & GGA & AGG & CGt & CAA & СCT & ATa & $\mathrm{CCa}$ & $\mathrm{AAa}$ & $\mathrm{GCa}$ \\
\hline ri & TCC & GAG & CAG & TCG & CAA & CCT & CGT & GGA & AGG & CGA & CAA & CCT & ATC & $\mathrm{CCC}$ & AAG & GCT \\
\hline & $\mathbf{R}$ & 0 & $D$ & $\mathbf{E}$ & $G$ & $\mathbf{R}$ & A & W & A & 0 & $=$ & G & $\mathbf{Y}$ & $\mathbf{P}$ & พ & $\mathbf{P}$ \\
\hline p & $\mathrm{aGg}$ & CAG & $\mathrm{CCt}$ & GAG & ; GGt & $\mathrm{AGa}$ & GCt & TGG & $\mathrm{GCa}$ & CAG & $\mathrm{CCa}$ & GGa & TAC & ССТ & TGG & $\mathrm{CCa}$ \\
\hline Dri & CGC & CAG & $\mathrm{CCC}$ & GAG & GGC & AGG & GCC & TGG & GCT & CAG & $\mathrm{CCT}$ & GGG & TAT & ССT & TGG & $\mathrm{CCC}$ \\
\hline & I & $\mathbf{Y}$ & G & $N$ & $F$ & G & $\mathbf{M}$ & G & W & A & G & W & L & I & $\mathbf{S}$ & $\mathbf{P}$ \\
\hline p & $\mathrm{tTg}$ & TAt & GGt & AAC & GAa & GGa & ATG & GGa & TGG & GCA & GGA & TGG & CTC & CTt & agc & $\mathrm{CCt}$ \\
\hline Ori & CTC & TAC & GGC & AAT & GAG & GGC & ATG & GGG & TGG & $\mathrm{GCA}$ & GGA & TGG & CTC & CTG & $T C A$ & $\mathrm{CCC}$ \\
\hline & $\mathbf{R}$ & G & r & $\mathbf{R}$ & $\mathbf{P}$ & $\mathbf{S}$ & พ & G & P & $T$ & D & $\mathbf{P}$ & $\mathbf{R}$ & $\mathbf{R}$ & $\mathbf{R}$ & $\mathbf{S}$ \\
\hline Dp & CGt & GGt & agt & $a G A$ & $\mathrm{CCa}$ & tcc & TGG & GGa & $\mathrm{cct}$ & $\mathrm{ACc}$ & GAt & $\mathrm{CCa}$ & $\mathrm{aGa}$ & $\mathrm{aGg}$ & cGt & TCt \\
\hline & CGC & GGC & TCT & CGA & СCT & AGT & TGG & GGC & $\mathrm{CCC}$ & $\mathrm{ACA}$ & GAC & $\mathrm{CCC}$ & CGG & CGT & AGG & TCG \\
\hline & $\mathbf{R}$ & $\mathbf{N}$ & I & 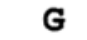 & K & K & D & E & L & & & & & & & \\
\hline
\end{tabular}

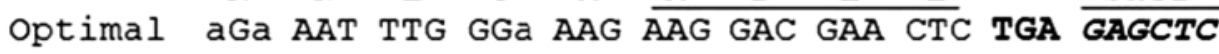

Original CGC AAT TTG GGT AAG

The changes to the original sequence are shown by lower case letters. Location of the Kozak sequence, 6 xHis-tag, nucleotides encoding KDEL and restriction sites for BamHI and SacI are indicated. ATG and TGA denote the start and termination codons, respectively.

3.2. Transformation of Agrobacterium tumefactions by Plant Expression Vectors

A. tumefactions strain LB4404 was transformed with PVXcore and $\mathrm{pBI}$-core vectors (Figure $3 \mathrm{~A}$ and $3 \mathrm{~B}$ ) in separate reactions via the standard freeze-thaw protocol (40). To this end, the HCVcp-coding plant constructs, pBI-core and PVX-core were introduced into Agrobacterium LB4404 (in the case of pBI-core) and into Agrobacterium LB4404; the latter had already harbored the helper plasmid pSOUP (41) (in the case of PVX-core), which is essential for replication of the PVX-GW-based plasmids in Agrobacterium species, respectively. Subsequently, transformed Agrobacterium cells were selected on plates containing $100 \mu \mathrm{g} /$ $\mathrm{mL}$ rifampicin (RIF) and $50 \mu \mathrm{g} / \mathrm{mL}$ kanamycin (KAN) and incubated for 72 hours at $28^{\circ} \mathrm{C}$. The transformed colonies were confirmed by gene-specific colony PCR using F-Kozak-VX and R-core-VX-specific primers, as described above. All the chemicals, culture media and antibiotics were purchased from Sigma (US) and Merck (Germany) companies.
3.3. Co-Agroinfiltration of Nicotiana tabacum Leaves by 19 Silencing-Suppressor Gene

To inhibit the silencing of foreign gene expression in tobacco, the constructed expression vectors were coinfiltrated with Agrobacterium strain LB4404, harboring the p19 silencing-suppressor gene (42) from tomato bushy stunt virus (TBSV) (GenBank accession number: M21958), which was previously cloned into pCAMBIA 1304 vector (CAMBIA Co., Australia) in our lab (P19pCAMBIA1304 vector). The agroinfiltration protocol was according to Kapila et al. study (43). In brief, the Agrobacterium cultures were first adjusted to OD 600 of 1.0 with MS medium supplemented with $2 \%$ (w/v) sucrose, $10 \mathrm{mM}$ MES $\mathrm{pH}=5.5,10 \mathrm{mM} \mathrm{MgCl}_{2}$, and induced for an additional 3 hours with $400 \mu \mathrm{M}$ acetosyringone. The Agrobacterium suspensions were subsequently mixed in equal ratios (half for p19 plasmid and half for each of the expression constructs, either PVX-core or pBI-core) and the N.tabacum (cultivar Jafarabadi; kindly provided by Dr. Tohidfar, Agricultural Biotechnology Research 
Institute of Iran) leaves were immersed in the bacterial suspension while a vacuum of 0.5 mbar was applied for two minutes. The infiltrated leaves were placed adaxial side down on wet Whatman paper in glass containers and subsequently incubated at $25^{\circ} \mathrm{C}$ with a 16 -hour photoperiod for five days.

\subsection{Protein Extraction}

The infiltrated tobacco leaves were ground into a fine powder in liquid nitrogen, using mortar and pestle. Subsequently, 0.5-mL extraction buffer ( $50 \mathrm{mM} \mathrm{NaH}_{2} \mathrm{PO}_{4}, \mathrm{pH}$ $=8,300 \mathrm{mM} \mathrm{NaCl}, 0.1 \%$ Tween 20, $10 \mathrm{mM}$ beta-mercaptoethanol, 1 mM PMSF, and 5 mM EDTA) was added to each gram of powdered leaf tissue. The total soluble protein (TSP) was removed from cell debris by centrifugation of the leaf extracts for 20 minutes at $4^{\circ} \mathrm{C}(14000 \mathrm{rpm})$.

The E. coli-derived HCVcp (eHCVcp) and plant-derived HCVcp (pHCVcp) were purified through application of nickel-nitrilotriacetic acid (Ni-NTA) chromatography, as previously described $(15,34)$. Protein concentrations were determined by Bradford assay (44) using bovine serum albumin (BSA) as standard.

\subsection{Sodium Dodecyl Sulfate Polyacrylamide Gel Electrophoresis and Blotting Analyses}

For dot blot analysis of proteins, equal concentration of TSP $(5 \mu \mathrm{g}-25 \mu \mathrm{g})$ from plant extracts, $5 \mu \mathrm{g}$ from eHCV as positive control and $25 \mu \mathrm{g}$ of non-agroinfiltrated plant extract were directly dotted on a nitrocellulose membrane. The membrane was dried at room temperature, blocked with 5\% (w/v) skim milk in PBS-Tween (1/1000 v/v), and after three washing steps incubated with anti-C/N terminal 5xHis antibody (Qiagen) 1:10000 in TBS-T for two hours at room temperature. Following the washing steps, the membrane was incubated with 1:8000 diluted horseradish peroxidase (HRP)-conjugated goat anti-mouse antibodies (Sigma, USA) for one hour. Finally, 3-3'diaminobenzidine (DAB, Sigma, USA) was used for color development. For SDS-PAGE and western blotting analyses, purified HCV core from E. coli and plant and plant crude extracts of the recombinant HCV core protein expressed by P19 coagroinfiltrated pBI121 and PVX vectors were loaded onto a 12\% SDS-polyacrylamide gel; by the end of electrophoresis, the protein bands were either stained with coomassie brilliant blue (Bio-Rad) or transferred to the PVDF membrane. Subsequently, the corresponding protein bands on the membrane were identified by biotinylated anticore polyclonal antibody (Abcam, UK) (1:1000 dilution) and streptavidin HRP conjugate (Sigma, USA) (1:4000 dilution), respectively, and were detected by TMB as substrate.

\subsection{Sandwich ELISA Analyses}

ELISA was performed using the streptavidin-biotin peroxidase complex (ABC) assay. Briefly, wells were coated overnight at room temperature with $100 \mu \mathrm{L}$ of $5 \mu \mathrm{g} / \mathrm{mL}$ mouse monoclonal antibody against 21-40 amino acids of HCV core antigen (Abcam, UK) and blocked with 1\% bovine serum albumin (BSA) for two hours at $37^{\circ} \mathrm{C}$. After several washing steps, $50 \mu \mathrm{g}$ of TSP from each sample or eHCVcp (for standard curve, serial dilutions in the range of $0.1-1 \mu \mathrm{g} / \mathrm{mL}$ ) were added to the coated wells in duplicates and incubated for one hour at $37^{\circ} \mathrm{C}$. Following the washing steps, biotinylated anti-core polyclonal antibodies (1:1000 dilution, Abcam, UK) were immersed into the wells and incubation continued at $37^{\circ} \mathrm{C}$ for one hour followed by washing steps and another similar incubation with streptavidin HRP conjugate (1:4000; Sigma). Finally, by adding TMB and color development, the absorbance at $450 \mathrm{~nm}$ was measured.

To detect the immuno-reactivity (antigenicity) of tobacco-derived HCVcp, an indirect ELISA for detection of anticore antibodies in human sera was developed as previously described (15). In brief, purified pHCVcp was coated in ELISA plates $(100 \mu \mathrm{L}$ of $10 \mu \mathrm{g} / \mathrm{mL})$. The pooled human sera (1/20 dilution) from five HCV seropositive patients and one serum negative (obtained from Tehran Blood Transfusion Center) were incubated with the plate for two hours at room temperature. HRP-labeled anti-human IgG (1:5000 dilution, Sigma, USA) was used for detection of bound antibodies and TMB was used for color development, as described above.

\subsection{Statistical Analyses}

Prism 5.0 software (GraphPad, USA) was used for data handling and statistical analysis was carried out using Mann-Whitney non-parametric and ANOVA tests. Statistical significance was set at $\mathrm{P} \leq 0.05$.

\section{Results}

\subsection{Codon Optimization of Tr-HCVcp for Efficient Expression in Plant}

Figure 1 represents the nucleotide changes applied in the basal sequence of HCVcp to improve its codon utilization for efficient expression in plants. Other elements (Kozak, KEDEL, His-tag and restriction sites) were also included in HCVcp basal sequence in Tr-HCVcp (Figure 1). In addition, to remove the plant mRNA destabilizing sequence "GGTAAG" (nucleotides 358-363) which is present in native HCVcp sequence, it was modified (Figure 1). As shown in Figure $2 \mathrm{~A}$, the corresponding nucleotide alterations increased the codon adaptation index (CAI) value from 0.65 to 0.85 and reduced the GC content from 62.62 to 51.05 (Figure 2 B). Results of DNA sequencing (not shown) indicated that the Kozak (GCCACCATGGC) sequence (36), harboring the start codon (ATG) at 5', KDEL nucleotides at $3^{-}$end, the designed restriction sites and all nucleotide modifications, was properly located in the Tr-HCVcp sequence (Figure 1). 
Figure 2. Distribution of Codon Usage Frequency Along the Length of the HCVcp Gene Sequence Before and After Optimization for Expression in Nicotiana tabacum

\section{A}

\section{Codon Adaptation Index (CAl)}
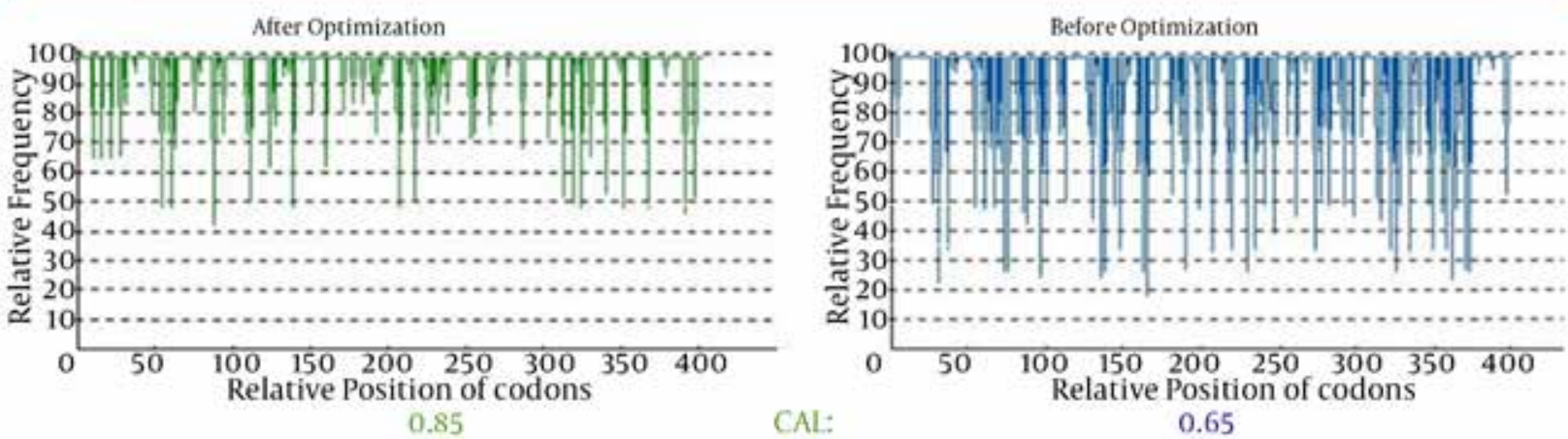

B

GCContent Adjustment
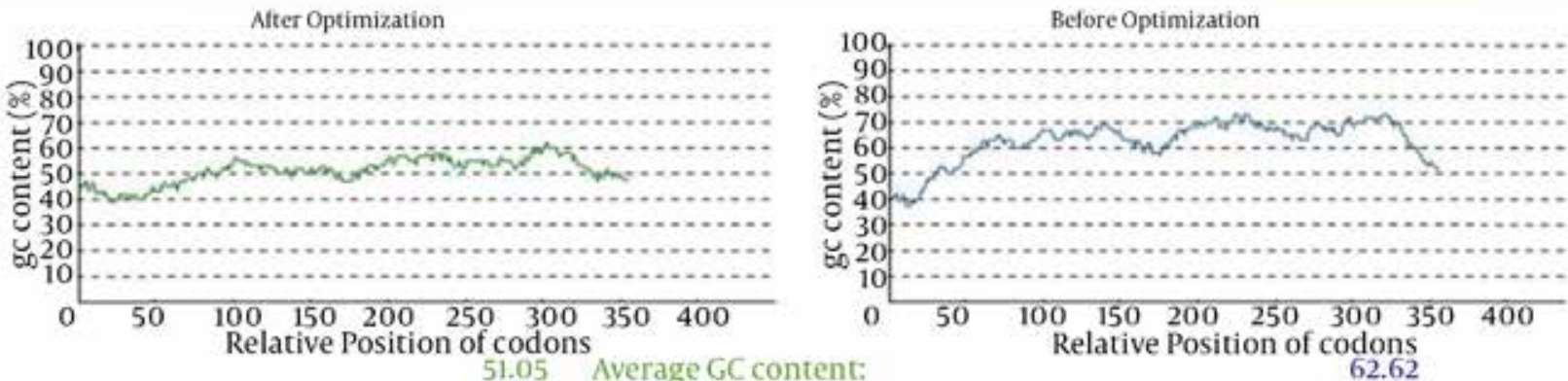

A) The corresponding nucleotide alterations increased the CAI value from 0.65 to 0.85 (the frequency of non-optimized bases decreased after optimization. The value of 100 is set for the codon with the highest usage frequency). B) The corresponding nucleotide alterations reduced the GC content from 62.62 to 51.05 .

\subsection{Tr-HCVcp Coding Plant Vectors}

Figure 3 represents the schematic of the constructed pBI-core (Figure $3 \mathrm{~A}$ ) and PVX-core (Figure $3 \mathrm{~B}$ ) plant expression vectors harboring the Tr-HCVcp, located under the control of CaMV 35S promoter (in pBI-core) and CP promoter (in PVX-core), upstream of the NOS-Ter transcriptional terminator. The sequencing results (not shown) confirmed the presence of the cloned Tr-HCVcp gene in pBI-core and PVX-core expression vectors with no alterations in the nucleotides. The resulting plasmids were transferred into A. tumefaciens LBA4404 target hosts (in the case of PVX-core, A. tumefaciens LBA4404 harboring the pSOUP vector). The transformed Agrobacteria were screened for recombinant constructs using core-specific primers (F-Kozak-VX and R-core-VX) and the colony PCR assay indicated the expected 439-bp fragments (Figure $3 \mathrm{C}$ ). One positive clone from each construct was subsequently used for agroinfiltration of $N$. tabacum leaves by p19 silencing-suppressor gene procedure as outlined in 3.3.

\subsection{Analyses of pHCVcp by Blotting Assays}

To detect and analyze pHCVcp, the transformed tobacco leave extracts were evaluated by dot and western blot assays (Figure 4). As shown in Figure $4 \mathrm{~A}$, dot blot analysis showed that HCV core was present in extracts of the tobacco leaves, transformed by each of the vectors (pBI-core and PVX-core), compared to negative controls with the same amount of loaded TSP. Although dot blot is a qualitative assay rather than a quantitative one, our result tentatively implied a higher level of expression for PVX-core compared with that of pBI-core construct, while the same amounts of TSP were applied (Figure $4 \mathrm{~A}$ ).

Coomassie-stained SDS-PAGE showed that nonspecifically-bound plant proteins were eluted from Ni-NTA affinity chromatography besides a band with an electrophoretic mobility of $15 \mathrm{kDa}$ (Figure $4 \mathrm{~B}$ ). The recent band 
A

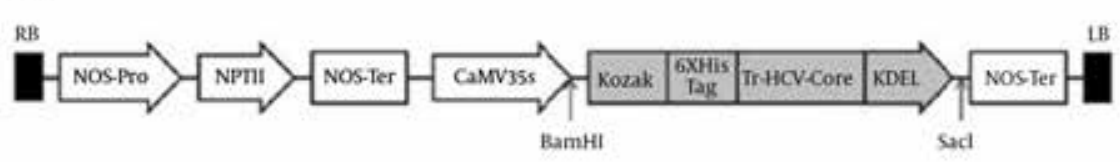

B

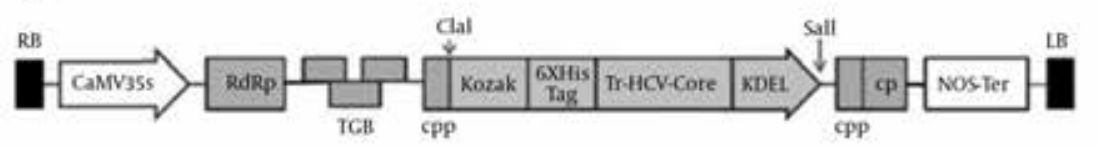

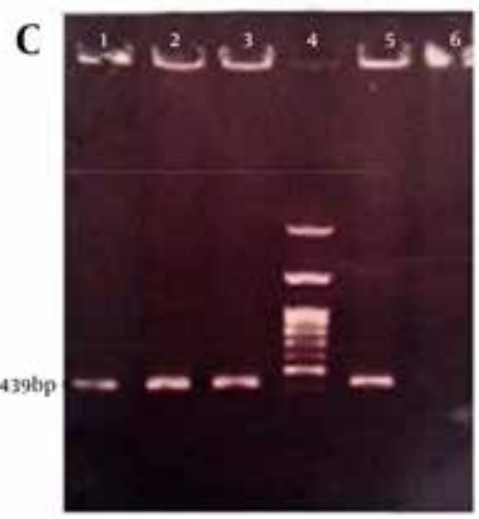

A) The synthetic Tr-HCVcp gene was inserted into pBI121 through BamHI/SacI sites under the control of CaMV 355 promoter. B) The Tr-HCVcp gene was cloned into ClaI/SalI sites of PVX-GW vector under the control of duplicated PVX-coat protein subgenomic promoter (CPP). C) Colony PCR analysis of transformed Agrobacterium LB4404. Lanes 1 and 2: PCR on colonies transformed with pBI-core; lanes 3 and 5: PCR on colonies transformed with PVX-core construct; lane 4:100-bp DNA ladder; lane 6, untransformed Agrobacterium (without constructs) as negative control. Appearance of the 439-bp fragment in transformed colonies indicated the positive colonies.

was visible on a Coomassie-stained SDS-PAGE gel (Figure $4 \mathrm{~B})$ and was also detected using anticore polyclonal antibodies in a western blot assay. There was no degradation of pHCVcp and the protein was not glycosylated because the size of the produced protein was as predicted by the protein sequence (https://www.genscript. com/ssl-bin/site2/peptide_calculation.cgi). As shown in Figure $4 \mathrm{C}$, the results of western blot analysis indicated that pHCVcp from PVX-core and pBI-core expressed a protein of approximately $15 \mathrm{kDa}$, while the negative control lacked the core protein and eHCVcp showed a higher molecular weight ( $21 \mathrm{kDa}$, which is due to addition of vector-derived amino acids in pIVEX2.4a plasmid) and a few other lower-molecular weight protein bands which were presumably the results of ribosomal release and uncompleted translation, as previously reported (15). It should be noted that dot blotting in this study was performed based on prior studies that used this procedure in complementation of western blotting $(7,8)$. Indeed, In western blotting, the protein will be completely denatured (by boiling in SDS-PAGE loading buffer which contains 2-ME and SDS) and therefore some information that might be related to the native/soluble structure of the protein might be lost (e.g. interaction of protein with a conformational antibody), while dot blot assay was used as a preliminary and fast test to show if the protein was expressed.

\subsection{Analysis of the Expression Levels of pHCVcp and Its Diagnostic Potency by ELISA}

The expression levels of pHCVcp by each of the vectors
(pBI-core as a classical plant binary vector and PVX-core as a viral-based vector) in the presence or absence of P-19 co-agroinfiltration were compared by ELISA. Sandwich ELISA is the assay of choice and is usual in quantification of antigen proteins (when an exact and precise standard curve is prepared and a specific antibody against the antigen is used) and a number of diagnostic ELISA kits work on this basis $(45,46)$. In this context and to perform an exact and precise quantitative ELISA, we used a specific monoclonal antibody against the core antigen (at the bottom of ELISA plates) and polyclonal anticore antibodies (at the top of the pHCVcp) in a sandwich ELISA-based protocol and also prepared a precise standard curve by applying serial dilution $(0.1-1 \mu \mathrm{g} / \mathrm{mL}$; data not shown) of purified eHCVcp to calculate the expression levels of pHCVcp. They were expressed in form of the percentage of TSP, which was determined using a Bradford protein assay. The leaves transformed by PVX (vector without HCVcp) and co-agroinfiltrated with P19 constructs were used as negative control (Figure $5 \mathrm{~A}$ ). The results indicated that the expression of pBI-core and PVX-core was around $0.004 \%$ of TSP in the absence of P19 co-agroinfiltration, whereas in its presence, the expression level of pBI-core and PVX-core increased to 0.019\% (210 ng/g) and $0.022 \%$ (240 ng/g) of TSP fresh leaf weight, respectively, which was almost five times improvement for pHCVcp expression (Figure $5 \mathrm{~A}$ ).

The diagnosis potency of pHCVcp for detection of HCVinfected human sera was also analyzed by ELISA. As shown in Figure 5 B, purified pHCVcp could detect HCV-infected sera in a comparable way to that of the eHCVcp. 
Figure 4. Sodium Dodecyl Sulfate Polyacrylamide Gel Electrophoresis and Blotting Analysis of HCVcp-Transient Expression in Tobacco Leaves

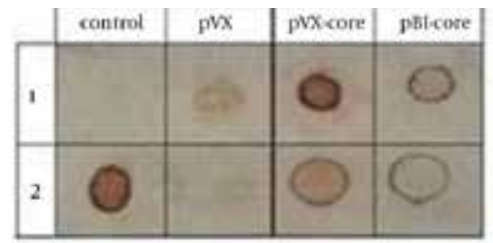

B

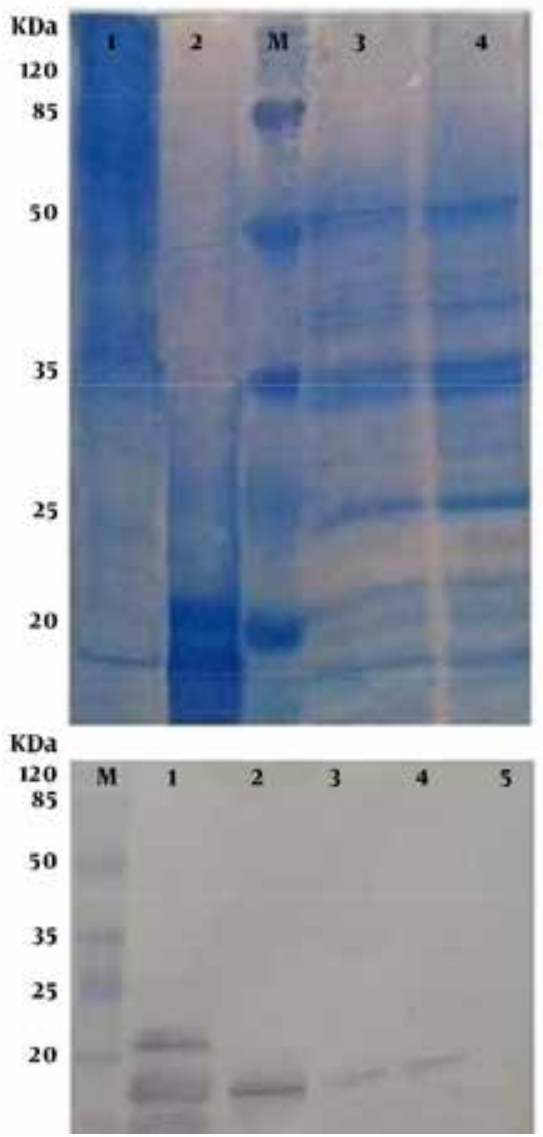

A). Dot blotting; at control column: row 1 : negative control ( $25 \mu \mathrm{g}$ TSP of untransformed tobacco leaves); row 2: $5 \mu \mathrm{g}$ positive control (eHCVcp). At PVX column: negative control (tobacco leaves transformed by PVX vector alone; ie, without Tr-HCVcp). PVX-core and pBI-core columns: the extracts from the PVX-core and pBI-core P19 co-agroinfiltrated leaves, respectively. Rows 2 and 1 in these two last columns correspond to $5 \mu \mathrm{g}$ and $25 \mu \mathrm{g}$ of TSP, respectively. B) Coomassie-stained 12\% SDS-PAGE gel, loaded with; lane 1: $5 \mu \mathrm{g}$ concentrated plant-purified HCVcp (from the PVX-core expression system). Lane 2: $5 \mu \mathrm{g}$ of purified eHCVcp. Lane 3: crude protein extract from agroinfiltrated leaves with PVX-core $(20 \mu \mathrm{g})$. Lane 4 : untransformed leaves $(20 \mu \mathrm{g})$. C) Western blotting; lane 1: positive control (700 ng purified eHCVcp). Lane 2: purified pHCVcp (700 ng from the PVX-core expression system). Lanes 3 and 4: the extracts from P19 co-agroinfiltrated PVXcore and pBI-core leaves, respectively ( $50 \mu \mathrm{g}$ of plant TSP was applied in each lane). Lane 5: negative control (50 $\mu \mathrm{g}$ of plant TSP of untransformed tobacco leaves). Lane M: prestained protein ladder (Fermentas). HCVcp denote to HCV core protein, eHCVcp and pHCVcp dnote to E.coli-derived and plant-derived $\mathrm{HCV}$ cp, respectively. In western blot and SDS-PAGE figures, the location of HCVcp under the $20 \mathrm{kDa}$ molecular weight range is shown by arrows. The reason for multiple bands in the case of eHCVcp is explained in the corresponding result section of the text. The results of SDS-PAGE showed that Ni-NTA pull-downs of plant extract contained endogenous nonspecific plant proteins besides pHCVcp. According to ELISA data, the concentration of pHCVcp was $1 / 20$ of the column-purified protein. Therefore, although $1 \mu \mathrm{g}(100 \mu \mathrm{L}$ of $10 \mu \mathrm{g} / \mathrm{mL}$ coated) was coated, only a small amount reacted (less than $50 \mathrm{ng}$ ) (Figure $5 \mathrm{~B}$ ). However, none of these endogenous nonspecific plant proteins reacted with anti-HCVcp in western blotting of the purified protein fraction.

\section{Discussion}

The primary objective of the present study was to provide an efficient transient tobacco system for expression of HCVcp in a regionally-adapted tobacco host (the Iranian Jafarabadi-tobacco plant cultivar). While other tobacco plant such as Australian Nicotiana species, N. benthamiana, is a well-known and hyper-susceptible host for plant-derived recombinant protein expression (47), to our knowledge there was no prior report available on expression in Jafarabadi tobacco cultivar (which is one of the currently available tobacco cultivar in Iran). To address this concern, the first strategy that should be considered would be the optimization of the gene sequence for efficient codon usage by the plant (27). In agreement, it is recently shown that the plant codonoptimized BPV-1 LI gene product increased significantly in comparison to the unmodified counterpart (28). As shown in Figure $2 \mathrm{~A}$, our codon optimization approach increased the CAI value from 0.65 to 0.85 , which was in favor of codon utilization for N. tabacum (35). Although increasing the CAI value is usually inevitably accompanied by increase of $A+T$ percentage (which enhances mRNA instability and decreases the efficiency of translation) $(48,49)$, our codon optimization approach that was based on the codon usage of nuclear-encoded genes of tobacco could keep the A + T percentage at $49 \%$, while reducing the GC content from 62.62 to 51.05 (Figure $2 \mathrm{~B}$ ), almost the perfect range desired for GC content (50\%). To ameliorate the expression level, insertion of the Kozak sequence (GCCACCATGGC) (36) at the translation start site (ATG) was also considered (Figure 1). However, since the two base pairs immediately following the ATG start codon were "C" and "A" and were not compatible with the optimized Kozak sequence (underlined nucleotides), consistent with the approach undertaken by Amani et al. (50), we had to insert the GCT sequence that codes for alanine (a nonpolar amino acid). Moreover, to improve the stability of pHCVcp, KDEL-encoded bases were also considered at the 3' site of the Tr-HCVcp sequence (Figure 1). Employing this strategy for expression of human epidermal growth factor in tobacco resulted in a $10^{4}$-fold higher yield of protein expression (51). Finally, in our codon optimization strategy, the possible deleterious splicing motif in plants "GGTAAG", resulting in RNA degradation and gene silencing of the gene (52), was removed from the native HCVcp-coding sequence (nucleotides 358-363).

In our study, two kinds of plant vectors were employed: a classic, nonreplicative, binary vector (pBI121) and a PVXbased viral replicative vector $(29,30)$. Our results indicated that the PVX-based vector provided higher expression levels of pHCVcp compared to that of pBI121 (Figure $4 \mathrm{~A}$ and $5 \mathrm{~A}$ ). Our result was in complete agreement with the results of a recent report comparing the expression levels by TMV and PVX replicative viral vectors which was three times of that of pBI121 vector (53). 
A

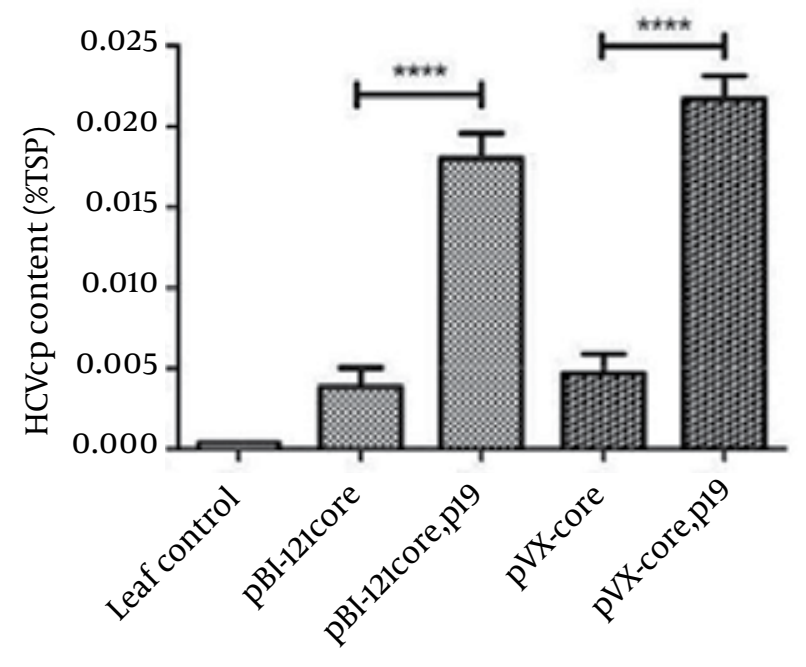

B

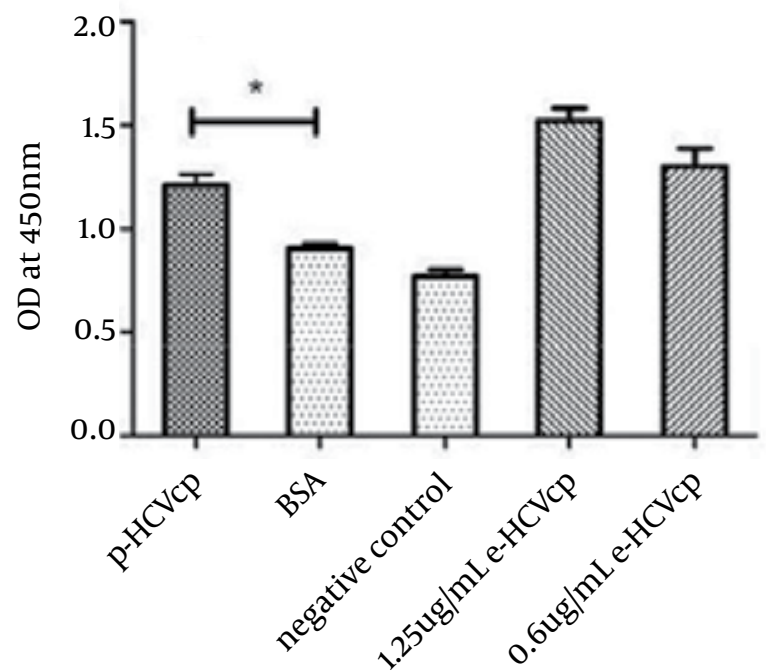

A) The expression level of pBI-core and PVX-core were assessed by ELISA and compared in the presence or absence of co-agroinfiltration by gene silencing suppressor P19 construct. Leaf control denotes the tobacco leaves transformed by PVX vector alone (ie, without Tr-HCVcP). B) Result of ELISA assay for confirmation of plant-derived HCVcp, using HCV-positive human serum. Negative control corresponds to HCV negative human sera. BSA was also used as another negative control. pHCVcp (corresponding to $100 \mu \mathrm{L}$ of $10 \mu \mathrm{g} / \mathrm{mL}$ of pHCVcp from PVX-core-transformed tobacco leaves) and eHCVcp denote plant-and E. coli-derived HCVcp, respectively. Statistical analysis was performed by one-way analysis of variance (ANOVA), using the Bonferroni's multiple comparison test.

For obtaining the highest expression levels, it is important to prevent the silencing mechanisms that limit foreign gene expressions in plants. It is shown that coexpression of viral silencing suppressor proteins eliminates the effects of post-transcriptional gene silencing (PTGS) in a transient expression system (42). Application of P19-mediated co-agroinfiltration has already shown to improve recombinant expression in plants by several folds (42). Our results indicated that the presence of P19 increased the expression levels of pHCVcp by five times in both pBI121 and PVX expression constructs (Figure $5 \mathrm{~A}$ ). Our data were consistent with recent reports on enhancement of the expression levels of SARS-CoV N (54), antibodies (53) and GFP (55) proteins in the presence of P19 by 3-5 and even 100 folds, respectively.

The 10 times differences in the transient expression levels of human growth hormone peptide by pBI121 in $N$. tabacum was $0.002 \%$ of TSP (56) to that of our study $(0.02 \%$ of TSP for pHCVcp). The enhanced expression levels in our study compared to this prior report should be a direct result of the codon optimization and gene silencing suppression strategies undertaken for Tr-HCVcp in our gene modification approaches.

Finally, the results of ELISA in our study (Figure 5) demonstrated that the transient expression of pHCVcp in tobacco leaves could provide the proper protein for diagnostic purposes and indicated its full functionality to react with poly/monoclonal anticore antibodies and
HCV infected human sera compared to that of eHCVcp (14). In addition, the proper conformation of pHCVcp to interact with specific monoclonal antibody against 2140 amino acids of the core antigen (coated at the bottom of the ELISA plates) and the polyclonal anticore antibodies (at the top of the pHCVcp in ELISA), supported the presence of various interactive epitopes on pHCVcp. The monoclonal antibody used in this study was against amino acids 21-40 on HCV core antigen. This region carries two important immunological epitopes which are conserved between HCV strains: 1a, 1b, and 2 (57): Moreover, the amino acids of 23-44 are highly promiscuous CD4+ T cell epitopes and the 35-44 amino acids are HCVspecific HLA-A2-restricted CTL epitopes. They have been shown to be effective immunogens in the vaccine phase of two clinical trials while are present on the HCV core particle or in multi-epitope complexes (58). Therefore, the primary results have proposed that the plant-produced pHCVcp might have the promising structure to be used as a vaccine candidate too. However, these primary results should be confirmed through animal and immunization studies.

Although these ending results were consistent with the prior two reports on appropriate antigenicity of tobaccoderived HCVcp, both of those previous studies addressed only transgenic (stable) tobacco-plant generations for $\operatorname{HCVcp}(21,22)$. However, transient expression in plants is currently the method of choice due to the simplicity and 
feasibility of rapid protein expression, omitting tissue culture and regeneration costs, and ease of large scale industrial plans growth (23-25). While transgenic (stable) plant generation needs longer periods of transgenic production and more deleterious effects on gene silencing (21) which is not encountered in transient-expression approach (59).

Taken together, the present study was the first (to our best of knowledge) to address construction of a transient tobacco expression system for HCVcp. For highest possible expression efficiency, the HCVcp DNA sequence was codon optimized (based on the CAI for nuclear-encoded genes of tobacco), destabilizing of the GGTAAG sequence in the native HCVcp coding sequence was altered and Kozak and KDEL sequences were included at the 5' and 3 ' ends, respectively. Two types of plant expression vectors (PVX-based and classic pBI121 binary) for HCVcp were constructed and their levels of protein expression in the presence and absence of P19 co-agroinfiltration were compared. The results indicated the significant effect of our approach in enhancement of the expression levels for pHCVcp. To our knowledge, all the above mentioned reports were first of their kinds for HCVcp and provided valuable information for plant-based expression studies on this antigen. The preliminary results (with five HCV positive sera) showed that plant-HCVcp has the capability to capture anti-HCVcp antibodies for potential clinical applications which should be further evaluated and confirmed by statistically acceptable numbers of positive sample sera. The next steps might be standardization of pHCVcp for application in diagnostic ELISA through application of positive sera with determined viral loads as well as precisely predetermined standard positive sera together with high number of optimization studies with different concentrations of antigen/antibody in ELISA with precise statistical analyses and evaluation of the pHCVcp for immunization studies.

\section{Acknowledgements}

It is with affection and appreciation that Dr. A. Jafari from the Molecular Biology Department of Pasteur Institute of Iran is acknowledged for her supports and help concerning this project

\section{Authors' Contributions}

The present study was in part to fulfill the thesis of Mrs. Sara Mohammadzadeh in Ph.D. S. M. the Ph.D. candidate; she was responsible for most experimental works and prepared the predraft of the manuscript. Alireza Khabiri helped with ELISA assays. Farzin Roohvand: advisor; helped with expression of eHCVcp, diagnosis potency of pHCVcp by ELISA and editing of the manuscript. Arash Memarnejadian: co-advisor; Soheila Ajdary and Ali Hatef Salmanian: co-supervisors; Parastoo Ehsani: supervisor; design of the experimental works and editing the manuscript.

\section{Funding/Support}

This work was supported by Pasteur Institute of Iran, Tehran, Iran.

\section{References}

1. Mirzazadeh A, Salimzadeh H, Arabi M, Navadeh S, Hajarizadeh B Haghdoost AA. Trends of Obesity in Iranian Adults from 1990s to late 2000s; a Systematic Review and Meta-analysis. Middle East J Dig Dis. 2013;5(3):151-7.

2. Chevaliez S, Pawlotsky JM. Virology of hepatitis C virus infection. Best Pract Res Clin Gastroenterol. 2012;26(4):381-9.

3. Fauvelle C, Lepiller Q, Felmlee DJ, Fofana I, Habersetzer F, StollKeller F, et al. Hepatitis $C$ virus vaccines--progress and perspectives. Microb Pathog. 2013;58:66-72.

4. Roohvand F, Kossari N. Advances in hepatitis $C$ virus vaccines, Part one: Advances in basic knowledge for hepatitis $C$ virus vaccine design. Expert Opin Ther Pat. 2011;21(12):1811-30.

5. Roohvand F, Kossari N. Advances in hepatitis $C$ virus vaccines, part two: advances in hepatitis $C$ virus vaccine formulations and modalities. Expert Opin Ther Pat. 2012;22(4):391-415.

6. Hartoonian C, Sepehrizadeh Z, Tabatabai Yazdi M, Jang YS, Langroudi L, Amir Kalvanagh P, et al. Enhancement of Immune Responses by Co-delivery of CCL19/MIP-3beta Chemokine Plasmid With HCV Core DNA/Protein Immunization. Hepat Mon. 2014;14(3).

7. Memarnejadian A, Roohvand F, Arashkia A, Rafati S, Shokrgozar MA. Polytope DNA vaccine development against hepatitis $C$ virus: a streamlined approach from in silico design to in vitro and primary in vivo analyses in BALB/c mice. Protein Pept Lett. 2009;16(7):842-50.

8. Arashkia A, Roohvand F, Memarnejadian A, Aghasadeghi MR Rafati S. Construction of HCV-polytope vaccine candidates harbouring immune-enhancer sequences and primary evaluation of their immunogenicity in BALB/c mice. Virus Genes. 2010;40(1):44-52.

9. Memarnejadian A, Roohvand F. Fusion of HBsAg and prime boosting augment Th1 and CTL responses to HCV polytope DNA vaccine. Cell Immunol. 2010;261(2):93-8.

10. Seme K, Poljak M, Babic DZ, Mocilnik T, Vince A. The role of core antigen detection in management of hepatitis $\mathrm{C}$ : a critical review. J Clin Virol. 2005;32(2):92-101.

11. Baghbani-arani F, Roohvand F, Aghasadeghi MR, Eidi A, Amini S, Motevalli F, et al. Expression and characterization of Escherichia coli derived hepatitis C virus ARFP/F protein. Mol Biol (Mosk). 2012;46(2):251-9.

12. Zhu W, Chang Y, Wu C, Han Q, Pei R, Lu M, et al. The wild-type hepatitis $C$ virus core inhibits initiation of antigen-specific $T$ - and B-cell immune responses in BALB/c mice. Clin Vaccine Immunol. 2010;17(7):1139-47.

13. Cerutti A, Maillard P, Minisini R, Vidalain PO, Roohvand F, Pecheur EI, et al. Identification of a functional, CRM-1-dependent nuclear export signal in hepatitis $\mathrm{C}$ virus core protein. PLoS One. 2011;6(10).

14. Mavromara P, Sall A, Kalinina O, Horm V, Budkowska A, H. C. V. Collaborative Team of the International Pasteur Network. The impact of HCV diversity on diagnosis tools for HCV infection. Med Mal Infect. 2005;35 Suppl 2:S103-4.

15. Roohvand F, Aghasadeghi MR, Sadat SM, Budkowska A, Khabiri AR. HCV core protein immunization with Montanide/CpG elicits strong Th1/Th2 and long-lived CTL responses. Biochem Biophys Res Commun. 2007;354(3):641-9.

16. Acosta-Rivero N, Duenas-Carrera S, Alvarez-Lajonchere L, Morales-Grillo J. HCV core protein-expressing DNA vaccine induces a strong class I-binding peptide DTH response in mice. Biochem Biophys Res Commun. 2004;314(3):781-6.

17. Yazdanian M, Memarnejadian A, Mahdavi M, Sadat SM, Moteval F, Vahabpour R, et al. Immunization of Mice by BCG Formulated HCV Core Protein Elicited Higher Th1-Oriented Responses Compared to Pluronic-F127 Copolymer. Hepat Mon. 2013;13(10).

18. Ohadi M, Rasouli R, Darzi-Eslam E, Jafari A, Ehsani P. Expression of 
Shigella flexneri ipaB Gene in Tobacco. Avicenna J Med Biotechnol. 2013;5(2):118-24

19. Guan ZJ, Guo B, Huo YL, Guan ZP, Wei YH. Overview of expression of hepatitis B surface antigen in transgenic plants. Vaccine. 2010;28(46):7351-62.

20. Lai H, Chen Q. Bioprocessing of plant-derived virus-like particles of Norwalk virus capsid protein under current Good Manufacture Practice regulations. Plant Cell Rep. 2012;31(3):573-84.

21. Nianiou I, Kalantidis K, Madesis P, Georgopoulou U, Mavromara P, Tsaftaris A. Expression of an HCV core antigen coding gene in tobacco (N. tabacum L.). Prep Biochem Biotechnol. 2008;38(4):411-21.

22. Madesis P, Osathanunkul M, Georgopoulou U, Gisby MF, Mudd EA, Nianiou I, et al. A hepatitis $C$ virus core polypeptide expressed in chloroplasts detects anti-core antibodies in infected human sera.J Biotechnol. 2010;145(4):377-86.

23. Meyers A, Chakauya E, Shephard E, Tanzer FL, Maclean J, Lynch A et al. Expression of HIV-1 antigens in plants as potential subunit vaccines. BMC Biotechnol. 2008;8:53.

24. Komarova TV, Baschieri S, Donini M, Marusic C, Benvenuto E, Dorokhov YL. Transient expression systems for plant-derived biopharmaceuticals. Expert Rev Vaccines. 2010;9(8):859-76.

25. Mett V, Musiychuk K, Bi H, Farrance CE, Horsey A, Ugulava N, et al. A plant-produced influenza subunit vaccine protects ferrets against virus challenge. Influenza Other Respir Viruses. 2008;2(1):33-40.

26. Shoji Y, Farrance CE, Bautista J, Bi H, Musiychuk K, Horsey A, et al. A plant-based system for rapid production of influenza vaccine antigens. Influenza Other Respir Viruses. 2012;6(3):204-10.

27. Perlak FJ, Fuchs RL, Dean DA, McPherson SL, Fischhoff DA Modification of the coding sequence enhances plant expression of insect control protein genes. Proc Natl Acad Sci U S A. 1991;88(8):3324-8.

28. Love AJ, Chapman SN, Matic S, Noris E, Lomonossoff GP, Taliansky M. In planta production of a candidate vaccine against bovine papillomavirus type 1. Planta. 2012;236(4):1305-13.

29. Gleba Y, Klimyuk V, Marillonnet S. Magnifection--a new platform for expressing recombinant vaccines in plants. Vaccine. 2005;23(17-18):2042-8.

30. Hefferon KL. Plant virus expression vectors set the stage as production platforms for biopharmaceutical proteins. Virology 2012;433(1):1-6.

31. Obembe OO, Popoola JO, Leelavathi S, Reddy SV. Advances in plant molecular farming. Biotechnol Adv. 2011;29(2):210-22.

32. Lacorte C, Ribeiro SG, Lohuis D, Goldbach R, Prins M. Potatovirus $\mathrm{X}$ and Tobacco mosaic virus-based vectors compatible with the Gateway cloning system. JVirol Methods. 2010;164(1-2):7-13.

33. Maillard P, Lavergne JP, Siberil S, Faure G, Roohvand F, Petres S, et al. Fcgamma receptor-like activity of hepatitis $\mathrm{C}$ virus core protein. JBiol Chem. 2004;279(4):2430-7.

34. Roohvand F, Maillard P, Lavergne JP, Boulant S, Walic M, Andreo U, et al. Initiation of hepatitis $C$ virus infection requires the dynamic microtubule network: role of the viral nucleocapsid protein. $J$ Biol Chem. 2009;284(20):13778-91.

35. Sharp PM, Li WH. The codon Adaptation Index-a measure of directional synonymous codon usage bias, and its potential applications. Nucleic Acids Res. 1987;15(3):1281-95.

36. Kozak M. The scanning model for translation: an update. J Cell Biol.1989;108(2):229-41.

37. Munro S, Pelham HR. A C-terminal signal prevents secretion of luminal ER proteins. Cell.1987;48(5):899-907.

38. Chen PY, Wang C, Soong S, To K. Complete sequence of the binary vector pBI121 and its application in cloning T-DNA insertion from transgenic plants. Molecular Breeding. 2003;11(4):287-93.

39. Sambrook J, Russell DW. Molecular cloning.New York: Cold Spring Harbor Laboratory; 2001.

40. Hofgen R, Willmitzer L. Storage of competent cells for Agrobacterium transformation. Nucleic Acids Res. 1988;16(20):9877.
41. Hellens RP, Edwards EA, Leyland NR, Bean S, Mullineaux PM pGreen: a versatile and flexible binary Ti vector for Agrobacterium-mediated plant transformation. Plant Mol Biol. 2000;42(6):819-32.

42. Voinnet O, Rivas S, Mestre P, Baulcombe D. An enhanced transient expression system in plants based on suppression of gene silencing by the p19 protein of tomato bushy stunt virus. Plant $J$ 2003;33(5):949-56.

43. Kapila J, De Rycke R, Van Montagu M, Angenon G. An Agrobacterium-mediated transient gene expression system for intact leaves. Plant Sci J.1997;122(1):101-8.

44. Bradford MM. A rapid and sensitive method for the quantitation of microgram quantities of protein utilizing the principle of protein-dye binding. Anal Biochem. 1976;72:248-54

45. Sharma N, Kim T, Yang M. Production and secretion of human interleukin-18 in transgenic tobacco cell suspension culture. Biotech Bio Engin. 2006;11(2):154-9.

46. Castilho A, Neumann L, Gattinger P, Strasser R, Vorauer-Uhl K, Sterovsky $\mathrm{T}$, et al. Generation of biologically active multi-sialylated recombinant human EPOFc in plants. PLoS One. 2013;8(1)

47. Goodin MM, Zaitlin D, Naidu RA, Lommel SA. Nicotiana benthamiana: its history and future as a model for plant-pathogen interactions. Mol Plant Microbe Interact. 2008;21(8):1015-26.

48. Suo G, Chen B, Zhang J, Duan Z, He Z, Yao W, et al. Effects of codon modification on human BMP2 gene expression in tobacco plants. Plant Cell Rep. 2006;25(7):689-97.

49. Laguia-Becher M, Martin V, Kraemer M, Corigliano M, Yacono ML, Goldman A, et al. Effect of codon optimization and subcellular targeting on Toxoplasma gondii antigen SAG1 expression in tobacco leaves to use in subcutaneous and oral immunization in mice. BMC Biotechnol. 2010;10:52.

50. Amani J, Mousavi SL, Rafati S, Salmanian AH. In silico analysis of chimeric espA, eae and tir fragments of Escherichia coli O157:H7 for oral immunogenic applications. Theor Biol Med Model. 2009;6:28.

51. Wirth S, Calamante G, Mentaberry A, Bussmann L, Lattanzi M, Barañao L, et al. Expression of active human epidermal growth factor (hEGF) in tobacco plants by integrative and non-integrative systems. Molecul Breeding J. 2004;13(1):23-35.

52. Brendel V, Kleffe J, Carle-Urioste JC, Walbot V. Prediction of splice sites in plant pre-mRNA from sequence properties. $J$ Mol Biol. 1998;276(1):85-104

53. Komarova TV, Kosorukov VS, Frolova OY, Petrunia IV, Skrypnik KA, Gleba YY, et al. Plant-made trastuzumab (herceptin) inhibits HER2/Neu+ cell proliferation and retards tumor growth. PLoS One. 2011;6(3).

54. Zheng N, Xia R, Yang C, Yin B, Li Y, Duan C, et al. Boosted expression of the SARS-CoV nucleocapsid protein in tobacco and its immunogenicity in mice. Vaccine. 2009;27(36):5001-7.

55. Komarova TV, Schwartz AM, Makarov AA, Dorokhov YL. A new viral vector exploiting RNA polymerase I-mediated transcription. Biochemistry (Mosc). 2012;77(5):532-8.

56. Sohi HH, Jourabchi E, Khodabandeh M. Transient expression of human growth hormone in potato (Solanum tuberosum), tobacco (Nicotiana tobacum) and lettuce (Lactuca sativa) leaves by agroinfiltration. Iranian J Biotech. 2005;3(2)

57. Torresi J, Johnson D, Wedemeyer H. Progress in the development of preventive and therapeutic vaccines for hepatitis $C$ virus. Hepatol. 2011;54(6):1273-85.

58. Firbas C, Boehm T, Buerger V, Schuller E, Sabarth N, Jilma B, et al. Immunogenicity and safety of different injection routes and schedules of IC41, a Hepatitis C virus (HCV) peptide vaccine. Vaccine. 2010;28(12):2397-407.

59. Peach C, Velten J. Transgene expression variability (position ef fect) of CAT and GUS reporter genes driven by linked divergent T-DNA promoters. Plant Mol Biol. 1991;17(1):49-60. 